Kuschel, Annett; Beslmeisl, Sandra; Hahlweg, Kurt; Hautzinger, Martin

\title{
17 Tipps wie man Kinder erzieht - Welche Erziehungsstrategien setzen Eltern
} ein?

Praxis der Kinderpsychologie und Kinderpsychiatrie 56 (2007) 8, S. 691-706

Quellenangabe/ Reference:

Kuschel, Annett; BesImeisl, Sandra; Hahlweg, Kurt; Hautzinger, Martin: 17 Tipps wie man Kinder erzieht - Welche Erziehungsstrategien setzen Eltern ein? - In: Praxis der Kinderpsychologie und

Kinderpsychiatrie 56 (2007) 8, S. 691-706 - URN: urn:nbn:de:0111-opus-30585 - DOI: 10.25656/01:3058

https://nbn-resolving.org/urn:nbn:de:0111-opus-30585

https://doi.org/10.25656/01:3058

in Kooperation mit / in cooperation with:

\section{Vandenhoeck \& Ruprecht}

\section{V\&R}

http://www.v-r.de

\section{Nutzungsbedingungen}

Gewährt wird ein nicht exklusives, nicht übertragbares, persönliches und beschränktes Recht auf Nutzung dieses Dokuments. Dieses Dokument ist ausschließlich für den persönlichen, nicht-kommerziellen Gebrauch bestimmt. Die Nutzung stellt keine Übertragung des Eigentumsrechts an diesem Dokument dar und gilt vorbehaltlich der folgenden Einschränkungen: Auf sämtlichen Kopien dieses Dokuments müssen alle Urheberrechtshinweise und sonstigen Hinweise auf gesetzlichen Schutz beibehalten werden. Sie dürfen dieses Dokument nicht in irgendeiner Weise abändern, noch dürfen Sie dieses Dokument für öffentliche oder kommerzielle Zwecke vervielfältigen, öffentlich ausstellen, aufführen, vertreiben oder anderweitig nutzen.

Mit der Verwendung dieses Dokuments erkennen Sie die Nutzungsbedingungen an.

\section{Terms of use}

We grant a non-exclusive, non-transferable, individual and limited right to using this document.

This document is solely intended for your personal, non-commercial use. Use of this document does not include any transfer of property rights and it is conditional to the following limitations: All of the copies of this documents must retain all copyright information and other information regarding legal protection. You are not allowed to alter this document in any way, to copy it for public or commercial purposes, to exhibit the document in public, to perform, distribute or otherwise use the document in public.

By using this particular document, you accept the above-stated conditions of use.

\section{Kontakt / Contact:}

\section{peDOcs}

DIPF | Leibniz-Institut für Bildungsforschung und Bildungsinformation

Informationszentrum (IZ) Bildung

E-Mail: pedocs@dipf.de

Internet: www.pedocs.de

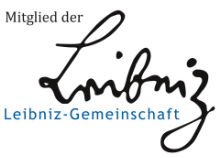




\title{
17 Tipps wie man Kinder erzieht - Welche Erziehungsstrategien setzen Eltern ein?
}

\author{
Annett Kuschel, Sandra Beslmeisl, Kurt Hahlweg und Martin Hautzinger
}

\section{Summary \\ 17 tips on parenting - which strategies do parents apply?}

The necessity to make available education support to parents is undisputed because of the epidemiology of child behavior problems. Parent trainings are a method to increase parenting competence. Triple $\mathrm{P}$ as a successful example of a parenting and family support program is introduced briefly with his theoretical bases, intervention levels, basic principles and education strategies. In addition, findings are reported to the effectiveness and consumer satisfaction. The present work examines which Triple P-strategies use parents after the training in the everyday life. The data come from the project "Zukunft Familie" in which the long-term effectiveness of the Triple P-training was examined in a controlled study in 280 families. 144 parents took part in the Triple P-group training and filled before, directly after the intervention and with 1-year-follow up questionnaires to the education strategies. The results show that the positive education strategies were used for promotiong child development already before the training more often as the ideas to managing misbehavior. More than $90 \%$ of the parents, above all, the positive education strategies also practised with the 1-year follow-up. In addition, there was a significant decrease by the use of the "time out"-strategy.

Prax. Kinderpsychol. Kinderpsychiat. 56/2007, 691-706

\section{Keywords}

universal prevention - Triple $\mathrm{P}$ - parental competence - education

\section{Zusammenfassung}

Die Notwendigkeit, Erziehungshilfen für Eltern zur Verfügung zu stellen, ist angesichts der Verbreitung von psychischen Störungen bei Kindern und Jugendlichen unbestritten. Elterntrainings sind eine verbreitete Maßnahme zur Steigerung der Erziehungskompetenz. Triple $P$ als erfolgreiches Beispiel für ein familien- und erziehungsunterstützendes Programm wird mit seinen theoretischen Grundlagen, Interventionsebenen, Grundprinzipien und Erziehungsstrategien vorgestellt. Darüber hinaus werden Befunde zur Wirksamkeit und Kurszufriedenheit berichtet. Die vorliegende Arbeit untersucht, welche der im Triple P-Gruppentraining vermittelten Erziehungsstrategien von den Eltern im Alltag umgesetzt werden. Die Daten stammen aus dem Projekt „Zukunft Familie“, in dem die längerfristige Wirksamkeit des Elterntrainings in einer kontrollierten Studie an 280 Familien untersucht wurde. Insgesamt nahmen 144 Eltern am Gruppentraining teil und füllten vor, direkt nach der Intervention 
und bei der 1-Jahres-Katamnese Kursbeurteilungs- bzw. Fragebogen zu den Erziehungsstrategien aus. Die Ergebnisse zeigen, dass die positiven Erziehungsstrategien zur Förderung der kindlichen Entwicklung bereits vor dem Training weiter verbreitet waren als die Tipps zum Umgang mit kindlichem Problemverhalten. Über 90 \% der Eltern praktizierten die positiven Erziehungsstrategien auch bei der 1-Jahres-Nachuntersuchung. Darüber hinaus gab es eine signifikante Abnahme bei der Verwendung der „Auszeit“- Strategie.

\section{Schlagwörter}

universelle Prävention - Triple P - Erziehungskompetenz - Erziehungsstrategien

\section{$1 \quad$ Einleitung}

Die mediale Präsenz von „Super-Nannys“ lässt auf ein großes Interesse an Erziehungsratgebern in der Bevölkerung schließen. Vielfach sind Eltern hinsichtlich der Erziehung ihrer Kinder verunsichert und erhoffen sich auf diese Weise Ratschläge oder Bestätigung. So gaben z. B. in einer Befragung an über 800 Eltern von Kindergartenkindern $68 \%$ an, dass sie nicht wissen, ob sie die Erziehungsaufgabe gut oder schlecht erfüllen. Nur 37 \% waren der Meinung, dass Erziehung zu schaffen ist und auftretende Probleme leicht zu lösen sind (Lübke et al., 2000). Auch in der wissenschaftlichen Diskussion sind die Themen Steigerung der Erziehungskompetenz und Ansätze zur Förderung der psychischen Gesundheit von Kindern in letzter Zeit immer stärker in den Vordergrund gerückt (Heinrichs, Hahlweg, Döpfner, 2006a; Petermann u. Petermann, 2006).

Die Notwendigkeit für die Prävention psychischer Störungen im Kindesalter ist in den letzten Jahren immer deutlicher geworden. Das U.S. Department of Health and Human Services hat in den 90er Jahren Ziele für die Reduktion von Prävalenz- und Inzidenzraten psychischer Auffälligkeiten aufgestellt (s. Greenberg, Domitrovich, Bumberger, 2001). Konkret sollte u. a. die Prävalenzrate psychischer Auffälligkeiten im Kindesalter von $20 \%$ auf weniger als $17 \%$ reduziert werden. Noch immer ist in den USA (wie auch in Deutschland) etwa jedes fünfte Kind von einer psychischen Störung betroffen (Kuschel et al., 2004). Meist handelt es sich hierbei um schwierige, chronische und kostenintensive Verhaltens- und emotionale Störungen, die auch mit deutlichen gesundheitlichen Beeinträchtigungen einhergehen. Eltern suchen jedoch nur selten professionelle Hilfe, selbst dann, wenn ihr Kind klinisch bedeutsame Symptome und Beeinträchtigungen aufweist (Offord et al., 1987).

Besonders solche Kinder sind einem erhöhten Risiko ausgesetzt, Verhaltens- und emotionale Probleme zu entwickeln, die keine enge, positive Beziehung zu den Eltern aufbauen konnten, strengen und inkonsequenten Erziehungsmaßnahmen ausgesetzt waren, deren Eltern Eheprobleme hatten, sich scheiden ließen oder bei denen ein Elternteil psychisch erkrankte. Anders ausgedrückt: „Kompetente Eltern haben auch kompetente Kinder“ (Schneewind, 1999, S. 139). Betont werden muss, dass die Beziehungen

(C) Vandenhoeck \& Ruprecht GmbH \& Co. KG, Göttingen 2007 
zwischen den Merkmalen des elterlichen Erziehungsverhaltens und den kindlichen Verhaltensstörungen nicht unidirektional, sondern transaktionaler Natur sind.

Die meisten Eltern werden auf ihre wichtige soziale Rolle kaum oder gar nicht vorbereitet. Sie können nur auf die in der eigenen Kindheit gemachten Erfahrungen zurückgreifen und lernen meist durch „Trial and Error“. In einer Gesellschaft, in der familiäre Netzwerke und Bande zerreißen, die Scheidungsraten hoch, alleinerziehende Elternteile und gemischte Familien alltäglich sind, benötigen Eltern mehr denn je Hilfe. Wir können es uns nicht leisten, die Bedürfnisse der Eltern nach mehr Unterstützung bei ihren erzieherischen Aufgaben zu ignorieren.

Im folgenden Abschnitt soll der Triple P-Ansatz als Beispiel für ein erfolgreiches erziehungs- und familienunterstützendes Programm näher vorgestellt werden. Dazu wird auf die theoretischen Grundlagen, die Interventionsebenen, Grundprinzipien und die Erziehungsstrategien eingegangen. Befunde zur Wirksamkeit und Kurszufriedenheit im deutschen Sprachraum schließen den Überblick ab.

\section{Triple $\mathrm{P}$ als Beispiel für ein erziehungs- und familienunterstützendes Programm}

Triple P steht für Positive Parenting Program und wurde in Australien von Sanders und Mitarbeitern an der Universität von Queensland in Brisbane am dortigen Parenting and Family Support Center als positives Erziehungsprogramm entwickelt (Sanders, 1999). Ziel dieses Programms ist es, Eltern Strategien nahe zu bringen, wie sie zu ihrem Kind eine positive Beziehung aufbauen, es in seiner Entwicklung fördern und wie sie effektiv mit problematischen Verhaltensweisen umgehen können. Durch Triple P wird die Eltern-Kind-Beziehung und das Erziehungsverhalten verbessert, das Kompetenzgefühl und die Bewältigungsstrategien der Eltern werden erhöht und emotionalen und Verhaltensproblemen von Kindern wird vorgebeugt. Es handelt sich bei Triple P um einen gesundheitspolitischen Ansatz mit Breitenwirkung, der multidisziplinär ausgerichtet ist.

Theoretischer Hintergrund: Triple P basiert auf dem aktuellen klinisch-psychologischen Forschungsstand und nimmt Bezug auf verschiedene theoretische Grundlagen wie z. B. Modelle sozialer Lerntheorie zur Eltern-Kind-Interaktion (Patterson, 1982), verhaltensanalytische Modelle, die besonders die Veränderungen der auslösenden Bedingungen von Problemverhalten thematisieren (Hart u. Risley, 1995), operante Lernprinzipien und die sozial-kognitive Lerntheorie nach Bandura (1977). Eine weitere wichtige theoretische Basis sind entwicklungspsychopathologische Befunde zu Risiko- und Schutzfaktoren für kindliche Verhaltensprobleme (Rutter, 1989). Besonders dysfunktionales Erziehungsverhalten, Stress, Ehekonflikte und unterschiedliche Auffassungen von Kindererziehung werden bei Triple P beachtet und stehen im Mittelpunkt verschiedener Interventionen.

Interventionsebenen: Ein Programm zur Unterstützung der Eltern sollte die Möglichkeit bieten, auf alle Bedürfnisse und Ressourcen der Familien individuell abgestuft 
einzugehen. Diesem Anspruch wird Triple P gerecht, da fünf Interventionsebenen jeweils eine steigende Intensität an Unterstützung beinhalten. Stufe 1 umfasst universelle Information über Erziehung, u. a. durch Medien und Informationsmaterialien (z. B. Zeitungskolumnen, Radiospots, Broschüre „Positive Erziehung“, „Kleine Helfer“; siehe www.triplep.de). Stufe 2 bietet Kurzberatungen für spezifische Erziehungsprobleme (Ess- oder Schlafprobleme, Wutanfälle, Trödeln) durch unterschiedliche Berufsgruppen mit Unterstützung von verschiedenen Materialien an. Zur Stufe 3 gehört neben der Kurzberatung ein aktives Training von bestimmten Erziehungsfertigkeiten. Die Stufe 4 umfasst ein intensives Elterntraining als Einzel- oder Gruppentraining oder als telefonisch unterstützte Selbstanleitung. Die Stufe 5 beinhaltet erweiterte verhaltenstherapeutische Interventionen auf der Familienebene für zusätzliche Schwierigkeiten wie Depression der Mütter, Partnerschaftskonflikte oder Substanzmissbrauch. Für eine detailliertere Darstellung der Inhalte und Ansatzpunkte des Programms siehe Hahlweg (2001) bzw. Sanders, Markie-Dadds und Turner (2003).

Prinzipien einer positiven Erziehung und Erziehungsstrategien von Triple P: Fünf allgemeine Prinzipien bilden die Grundlage der positiven Erziehung. Sie beziehen sich auf bekannte Schutz- und Risikofaktoren für kindliche Verhaltensprobleme und beschreiben zentrales Erziehungsverhalten (Cina et al., 2006; Sanders, 1996):

- Für eine sichere und interessante Umgebung sorgen. Kinder jeden Alters benötigen für eine gesunde Entwicklung Beaufsichtigung und ein sicheres Umfeld, welches ihnen Möglichkeiten zum Erkunden, Experimentieren und Spielen bietet. Dieses Prinzip ist unter Berücksichtigung des jeweiligen Entwicklungskontextes auch für ältere Kinder und Jugendliche von Bedeutung.

- Eine positive und anregende Lernatmosphäre schaffen. Die Eltern werden in ihrer Rolle als wichtige Lehrperson ihres Kindes ausgebildet. Das Programm zielt v. a. auf die Förderung der Problemlösefähigkeit der Kinder ab z. B. durch beiläufiges Lernen in verschiedenen Alltagssituationen (bei Bitten um Hilfe, Information, Ratschlag, Aufmerksamkeit).

- Sich konsequent verhalten. Die Eltern lernen die Grundlagen konsequenten Verhaltens kennen. Dies umfasst, Grundregeln für spezifische Situationen festzulegen, Regeln mit Kindern zu diskutieren, klare, ruhige, dem Alter angepasste Anweisungen zu geben oder (wenn nötig) absichtliches Ignorieren, logische Konsequenzen, den Stillen Stuhl oder die Auszeit einzusetzen. Diese Strategien stellen wirksame positive Alternativen zu Zwangsprozessen oder wirkungslosen Disziplinierungsmaßnahmen dar.

- Realistische Erwartungen haben. Die Eltern erhalten die Möglichkeit, ihre Erwartungen, Annahmen und Meinungen, mit denen sie das kindliche Verhalten erklären, kritisch zu hinterfragen und sich Ziele auszuwählen, die einerseits der kindlichen Entwicklung angemessen und andererseits realistisch sind.

- Die eigenen Bedürfnisse beachten. Elternschaft wird von verschiedenen Faktoren, die Auswirkungen auf die elterliche Selbstachtung und das Wohlbefinden 
haben, beeinflusst. Alle Triple P-Ebenen ermutigen Eltern dazu, dem eigenen Wohlbefinden und den eigenen Bedürfnissen Rechnung zu tragen und vermitteln ihnen die dafür erforderlichen Erziehungsstrategien.

Diese fünf allgemeinen Prinzipien werden in verschiedenen Erziehungsstrategien und -fertigkeiten auf den einzelnen Interventionsstufen umgesetzt. In Tabelle 1 sind die Inhalte des Triple P-Gruppentrainings der Ebene 4 sowie die vermittelten Erziehungsstrategien exemplarisch dargestellt.

Tab. 1: Übersicht über die Inhalte des Triple P-Gruppenprogramms und die 17 Erziehungsstrategien $(>)$.

\begin{tabular}{|c|c|}
\hline Sitzung & Themen und Erziehungsstrategien \\
\hline Sitzung 1: & Als Gruppe arbeiten \\
\hline \multirow[t]{4}{*}{ Positive Erziehung } & Was ist positive Erziehung? (5 Prinzipien) \\
\hline & Ursachen für Verhaltensprobleme \\
\hline & Ziele für Veränderungen \\
\hline & Kindliches Verhalten genau beobachten \\
\hline \multirow{13}{*}{$\begin{array}{l}\text { Sitzung 2: } \\
\text { Förderung der kindlichen Entwicklung }\end{array}$} & Entwicklung einer positiven Beziehungen zum Kind \\
\hline & $>$ Wertvolle Zeit \\
\hline & $>\quad$ Mit Kind sprechen \\
\hline & $>$ Zuneigung zeigen \\
\hline & Förderung angemessenen Verhaltens \\
\hline & $>$ Beschreibendes Lob \\
\hline & $>$ Aufmerksamkeit schenken \\
\hline & $>$ Spannende Aktivitäten \\
\hline & Beibringen neuer Fertigkeiten und Verhaltenweisen \\
\hline & $>$ Lernen am Modell \\
\hline & $>$ Beiläufiges Lernen \\
\hline & $>$ Fragen-Sagen-Tun \\
\hline & $>$ Punktekarte \\
\hline \multirow{11}{*}{$\begin{array}{l}\text { Sitzung 3: } \\
\text { Umgang mit Problemverhalten }\end{array}$} & Umgang mit Problemverhalten \\
\hline & $>$ Familienregeln \\
\hline & $>$ Direktes Ansprechen \\
\hline & $>$ Absichtliches Ignorieren \\
\hline & $>$ Klare, ruhige Anweisungen \\
\hline & $>$ Logische Konsequenzen \\
\hline & $>$ Stiller Stuhl \\
\hline & $>$ Auszeit \\
\hline & Entwicklung von Erziehungsroutinen \\
\hline & - Kooperationsroutine \\
\hline & - $\quad$ Routine zur Verhaltensänderung \\
\hline \multirow{3}{*}{$\begin{array}{l}\text { Sitzung 4: } \\
\text { Vorausplanen }\end{array}$} & „Überlebenstipps“ für Familien \\
\hline & Risikosituationen \\
\hline & - $\quad$ Aktivitätenpläne \\
\hline \multirow{2}{*}{$\begin{array}{l}\text { Sitzung 5-8: } \\
\text { Telefonkontakte }\end{array}$} & Besprechung von Fragen und Problemen \\
\hline & Motivation für die Umsetzung der erlernten Inhalte \\
\hline
\end{tabular}


Wirksamkeit: Wie eine Übersicht von Sanders (2006) zeigt, ist die empirische Evidenz insbesondere für das Triple P-Elterntraining sehr hoch. Es wurde in 29 randomisierten Kontrollgruppenstudien und 11 Einzelfallstudien erfolgreich empirisch überprüft. Zudem wurde in 17 weiteren Untersuchungen nachgewiesen, dass das Triple P-Elterntraining auch bei verschiedenen Risikogruppen (z. B. Kinder mit Entwicklungsstörungen, depressive Mütter) wirksam ist. Die mittlere Effektstärke der Studien liegt für expansives kindliches Problemverhalten bei 0,92. In den kontrollierten Studien gab es folgende signifikante Veränderungen: Reduktion des kindlichen Problemverhaltens, Verbesserung der elterlichen Erziehungskompetenz, Reduktion der psychischen Belastung der Eltern und Verbesserung der Partnerschaftsqualität. Das Triple P-Programm ist international mittlerweile in ca. 15 Ländern verbreitet. Zurzeit wird es in mehreren groß angelegten Public Health-Studien (z. B. Cina et al., 2006; Prinz u. Sanders, 2006) untersucht.

Für den deutschen Sprachraum legen Heinrichs et al. (2006b) Ergebnisse zur Wirksamkeit des Triple P-Gruppenprogramms als universelle Präventionsmaßnahme aus der Sicht von 219 Müttern und Vätern vor. Bei den Müttern zeigten sich signifikante Verbesserungen ihres Erziehungsverhaltens sowie deutliche Reduktionen des kindlichen Problemverhaltens. Darüber hinaus verringerte sich die psychische Belastung und die partnerschaftliche Zufriedenheit stieg an. Auch bei den Vätern zeigte sich eine Verbesserung des Erziehungsverhaltens. Es gab keine Anzeichen für eine differenzielle Wirksamkeit des Trainings für Jungen oder Mädchen bzw. für bestimmte Altersstufen. Die Analysen zur längerfristigen Wirksamkeit zeigen, dass für die Mütter die Effekte z. T. auch nach 2 und 3 Jahren bestehen bleiben. Aus Sicht der Väter waren kurzfristig nur wenige Veränderungen feststellbar. Jedoch konnte für das dysfunktionale Erziehungsverhalten eine Reduktion beobachtet werden. Dieser Unterschied bleibt bis zum Messzeitpunkt FU3 erhalten (Hahlweg et al., 2007). In einer nicht-randomisierten Interventionsstudie mit 197 sozial benachteiligten Familien von Kindergartenkindern zeigten sich deutliche Effekte, die mit denen der ersten Studie vergleichbar sind und auch ein Jahr später noch Bestand hatten (Heinrichs, 2006; Heinrichs, Krüger, Guse, 2006).

Kurszufriedenheit: In einer Studie von Heinrichs et al. (2006c) wird über die Teilnahmerate und Zufriedenheit als Indikatoren für die Akzeptanz und Einsetzbarkeit des Triple P-Elterntrainings im deutschen Sprachraum berichtet. An dieser Erhebung nahmen 341 Familien von Kindergartenkindern aus verschiedenen Einkommens- und Bildungsschichten teil. Es zeigten sich eine sehr hohe Teilnahmerate sowie eine hohe Zufriedenheit mit dem Training, unabhängig von soziodemographischen Merkmalen. Es ließen sich keine Belege dafür finden, dass das Triple P-Elterntraining nur für Eltern verhaltensauffälliger Kinder oder sozial begünstigte Familien geeignet wäre.

Cina et al. (2006) stellen in ihrem Beitrag ebenfalls Befunde zur Zufriedenheit und Akzeptanz vor. Dabei konnte auf Daten einer anfallenden Stichprobe von 1.695 Eltern aus Deutschland und der Schweiz zurückgegriffen werden. Die Ergebnisse zeigen, dass das Triple P-Programm bei den Eltern sehr gut ankommt und sie angeben, in hohem Maße davon profitiert zu haben. So sind $95 \%$ mit dem Programm zufrieden bis sehr 
zufrieden und $94 \%$ bewerteten die Qualität als gut bis hervorragend. Die in den Triple P-Kursen vermittelten Erziehungsstrategien wurden von 97 \% der Eltern als hilfreich bzw. sehr hilfreich für die Gestaltung des Erziehungsalltags erlebt.

Auszeit: In einer kritischen Stellungnahme zu Triple P äußern Deegener und Hurrelmann (2002), dass Triple P einen inflationären Gebrauch von Auszeitverfahren propagieren würde. In diesem Zusammenhang erscheint es wichtig, auf die z. Zt. verwendeten Erziehungspraktiken bei Vorschulkindern hinzuweisen. Manchmal entsteht bei der Diskussion hinsichtlich des elterlichen Umgangs mit problematischem (bes. aggressivem) Verhalten der Kinder der Eindruck, als ob die vermittelten Kompetenzen und Regeln die Eltern erst darauf bringen würden, Auszeitverfahren anzuwenden. Das Gegenteil ist der Fall, da Eltern sehr häufig andere, zum Teil sehr problematische und entwicklungshinderliche Maßnahmen anwenden.

So ist körperliche Bestrafung $(\mathrm{kB})$ bei Vorschulkindern noch weit verbreitet. In der später beschriebenen DFG-Studie gaben $73 \%$ der 280 untersuchten Mütter an, kB anzuwenden, wobei der überwiegende Teil kB gelegentlich (46 \%) bzw. in moderatem Ausmaß (13\%) verwendete; ca. $13 \%$ wendeten kB oft bzw. sehr oft an (Hahlweg et al., in Druck). Ähnlich sind auch die Ergebnisse anderer Studien: in der BMFSFJStudie (2003) verzichteten nur $28 \%$ der Eltern auf physische Strafe, 54 \% verwendeten leichtere und 17 \% schwere Körperstrafen. Perrez und Moggi (1990) ermittelten für Schweizer Mütter mit Kindern unter 12 Jahren eine Prävalenzrate von 80 \%, 55 \% gaben an, ihr Kind in den letzten 4 Wochen körperlich bestraft zu haben.

Körperliche Gewalt ist nicht die einzige Bestrafung, die Eltern anwenden. Psychische Sanktionen wie Fernsehverbot (71\%), Ausgehverbot (68\%), das Kind niederbrüllen (57\%), Taschengeldkürzung (38\%) und nicht mehr mit dem Kind reden (37\%) sind weit verbreitet (BMFSFJ, 2003). Die Befragung von Braunschweiger Eltern von Kindergartenkindern (Lübke et al., 2000) ergab, dass insgesamt $92 \%$ der Eltern Maßnahmen wie Aufs-Zimmer-Schicken oder Stubenarrest anwenden, $25 \%$ weniger als einmal im Monat und 67 \% 2- bis 3-mal im Monat bis täglich.

Ziel kognitiv-verhaltenstherapeutischer Elterntrainings ist es, durch die Vermittlung positiver Strategien die üblicherweise von Eltern praktizierten unangemessenen Maßnahmen zu reduzieren und durch angemessene Strategien zu ersetzen.

\section{Fragestellungen}

Im vorliegenden Beitrag wird dargestellt, a) wie häufig die einzelnen Erziehungsstrategien nach Beendigung des Triple P-Gruppenprogramms und bei der 1-JahresNachuntersuchung (FU1) angewendet werden; b) ob es einen Unterschied bezüglich der Rangfolge und Anwendungshäufigkeit von Erziehungsstrategien zwischen den Erhebungen unmittelbar nach dem Training und zu FU1 gibt; c) in welchen Situationen Eltern die Strategie „Auszeit“ anwenden und d) ob es Unterschiede bei der Anwendung der „Auszeit“ über die Messzeitpunkte gibt.

(C) Vandenhoeck \& Ruprecht GmbH \& Co. KG, Göttingen 2007 


\section{Methode}

\subsection{Design ${ }^{1}$ und Rekrutierung}

Die Rekrutierung erfolgte über die städtischen Kindertagesstätten in Braunschweig (Kitas). Der Ablauf und die Ergebnisse dieser Rekrutierung wurden im Detail bereits an anderer Stelle berichtet (Bertram et al., 2003; Heinrichs et al., 2005). Daher erfolgt hier nur eine kurze Zusammenfassung. 17 zufällig ausgewählte Kitas wurden zuerst hinsichtlich des Sozialstrukturindex (niedrig, mittel, gehoben) und der Anzahl der Kinder parallelisiert. Der objektive Kita-Sozialstrukturindex (OKS) ist ein $\mathrm{Maß}$ für die soziodemographische Struktur des Einzugsbereiches einer Kita. Er wurde auf Grund objektiver Daten (z. B. Arbeitslosenquote, Ausländeranteil) bestimmt. Im Anschluss wurden die parallelisierten Einrichtungen randomisiert und der Experimental- (EG) oder Kontrollgruppe zugeordnet. Die Datenerhebungen erfolgen zu sechs Messzeitpunkten: Prä (Eingangsdiagnostik), Post (direkt nach Beendigung des Trainings), Follow up 1 (nach 1 Jahr) bis zu Follow up 4 (nach 4 Jahren). Die vorliegende Studie berichtet über die Ergebnisse der Messungen Post und FU1.

Kriterien für die Teilnahme der Familien am Projekt waren das Alter des Kindes (2;6 bis 6 Jahre) sowie die Betreuung des Kindes in der Kita. Nicht berücksichtigt wurden Hort- und Krippenkinder unter 21/2 Jahren, Geschwisterkinder bereits angemeldeter Kinder sowie ausländische Familien mit deutlichen Problemen im Umgang mit der deutschen Sprache. Insgesamt wurden N = 280 Familien (populationsbezogene Rekrutierungsrate: 31 \%) für die Studie rekrutiert (Heinrichs et al., 2005).

\subsection{Studienstichprobe}

Insgesamt nahmen 144 Familien an dem Triple P-Gruppenprogramm teil. Es beteiligten sich 129 Eltern (90,2 \%) an der postalischen Datenerhebung unmittelbar nach dem Training (Post). Zur 1-Jahres-Nacherhebung lagen von $\mathrm{N}=111$ Familien (77,6 \%) vollständige Daten zum Einsatz der Triple P-Strategien vor.

Die EG setzte sich aus 68 Mädchen und 76 Jungen (53\%) zusammen. Das Alter betrug 4,5 Jahre $(S D=1.0) .21 \%$ der Kinder waren Einzelkinder und $56 \%$ hatten ein Geschwisterkind. Etwa jedes zehnte Kind wuchs bei einer allein erziehenden Mutter auf. Die Mütter waren durchschnittlich 35,5 Jahre $(S D=4.4)$, die Väter 38,8 Jahre $(S D=5.2)$ alt. 58,7 \% der Mütter und 65,6 \% der Väter hatten einen Hoch-

\footnotetext{
${ }^{1}$ Die Studie wurde durch die Deutsche Forschungsgemeinschaft (DFG, Projekt HA 1400/14-1) finanziell gefördert. Wir bedanken uns insbesondere bei den beteiligten Familien für ihr großes Engagement und bei Frau Joswig-Gröttrup und Frau Hamilton Kohn, Jugendamt der Stadt Braunschweig, Abteilung Kindertagesstätten, sowie bei allen Mitarbeitern der beteiligten städtischen Kindertagesstätten für die gute Zusammenarbeit und Kooperation. Dank geht auch an die früheren Mitarbeiterinnen des Projekts, Dr. Y. Kessemeier, Dr. H. Saßmann und an alle studentischen Hilfskräfte.
} 
schulabschluss. Etwa die Hälfte aller Familien verfügte über ein monatliches Haushaltseinkommen zwischen 1.500 und $3.000 €$, während etwa $35 \%$ ein Einkommen über $3.000 €$ zur Verfügung stand. $11 \%$ der Familien hatten einen Migranten-Status. Insgesamt handelte es sich um eine Stichprobe aus der mittleren bzw. oberen Sozialschicht, die nicht als repräsentativ für die Bevölkerung gelten kann, aber typisch für universelle Präventionsstudien ist (Lösel et al., 2005).

\subsection{Erhebungsinstrumente}

Die soziodemographischen Daten wurden in einem Interview erfasst, das zur Eingangsdiagnostik im Rahmen eines Hausbesuchs durchgeführt wurde. Mithilfe eines vorstrukturierten Fragebogens erhoben die Projekt-Mitarbeiter eine Vielzahl von Informationen. Es wurden Angaben zum Kind (Geschlecht, Alter, hauptsächlicher Aufenthaltsort) und zu den Eltern (Familienstand, Alter, Staatsangehörigkeit, Schulbildung, Beruf, Religion) erfragt.

Der Kursbeurteilungsfragebogen (KBF) wurde nach Beendigung des Gruppenprogramms (Post) von den Eltern ausgefüllt. Insgesamt besteht der Fragebogen aus 64 Items. Anhand der ersten 14 Items konnten die Eltern auf einer siebenstufigen Ratingskala $(1=$,sehr schlecht" bis $7=$ „hervorragend“) verschiedene Aspekte des Programms (z. B. Qualität, Kursleitung, Kursatmosphäre) einschätzen. Anschließend wurde erhoben, ob sich bereits Veränderungen gezeigt hatten.

Die Items 15 bis 59 lassen sich in zwei Kategorien einteilen. Zum einen sollten die Eltern anhand einer vierstufigen Ratingskala $(1=$ „stimmt gar nicht“ bis $4=$,stimmt vollkommen") beurteilen, wie hilfreich die einzelnen Sitzungen und Strategien waren. Zum anderen sollten die Eltern Angaben zur Anwendung der Strategien machen. Hier konnten sie folgende Antwortmöglichkeiten wählen ( 1 = „habe ich nie ausprobiert“', 2 $=$ "habe ich schon vorher praktiziert", 3 = ,habe ich im Rahmen des Programms ausprobiert", $4=$, habe ich als festen Bestandteil aufgenommen"). Abschließend erfassten zwei Items auf einer achtstufigen Ratingskala (von „nie“ bis „mehrmals täglich“), wie oft die Eltern aktuell die Auszeit anwenden und wie oft sie auszeitähnliche Verfahren vor der Intervention verwendet hatten. Bei Item 62 (offene Frage) notierten die Eltern Situationen, in denen sie die Auszeit eingesetzt hatten.

Zum Messzeitpunkt FU1 wurde der Triple P-Strategiefragebogen eingesetzt. Mittels einer 5-stufigen Ratingskala (von „nie“ bis „sehr oft“) schätzten die Eltern ein, wie häufig die einzelnen Strategien des Triple P-Gruppenprogramms in den vergangenen zwei Monaten angewendet wurden.

\subsection{Intervention}

Als Intervention wurde das Elterntraining Triple P im Gruppenformat (Stufe 4) durchgeführt. Den Eltern wird in vier je zweistündigen Sitzungen ein umfassendes Angebot verschiedener, wissenschaftlich erprobter Erziehungsstrategien vermittelt (s. Tab. 1).

(c) Vandenhoeck \& Ruprecht GmbH \& Co. KG, Göttingen 2007 
Zu den Sitzungen gehören kurze didaktische Präsentationen, Gruppendiskussionen, Erfahrungsaustausch und Unterstützung durch die Teilnehmer, das Demonstrieren der Erziehungsstrategien anhand kurzer Videosequenzen aus dem Video „Überlebenshilfe für Eltern" und das Einüben bestimmter Fertigkeiten im Rollenspiel. Zwischen den Sitzungen sollen die Eltern die Erziehungsstrategien zu Hause ausprobieren und üben. Begleitend zum Programm gibt es das „Gruppenarbeitsbuch für Eltern“. Im Anschluss an die Gruppensitzungen haben die Eltern die Möglichkeit, vier wöchentliche, individuelle Telefonkontakte von jeweils 15-20 Minuten Dauer wahrzunehmen, in denen Fortschritte, Fragen und auftretende Schwierigkeiten mit dem Trainer diskutiert werden können. Dies dient der Stabilisierung der Erziehungsstrategien und unterstützt die Generalisierung auf zukünftig auftretende Probleme. 23 \% der Teilnehmer verzichteten auf die optionalen Trainerkontakte, $39 \%$ nahmen alle vier Kontakte in Anspruch. Einen, zwei oder drei Kontakte nahmen jeweils 12-13\% der Eltern in Anspruch.

Fünf lizenzierte Trainer führten insgesamt 28 Triple P-Gruppen durch. Die Integrität der Durchführung wurde durch ein ausführliches Trainermanual sichergestellt. Außerdem wurden ca. 50 \% aller Gruppensitzungen von studentischen Hilfskräften protokolliert, um zu überprüfen, ob die Inhalte denen im Manual entsprechen. Die prozentuale Übereinstimmung lag für alle Sitzungen bei über 90 \%. Die Anwesenheit wurde von den Trainern dokumentiert. An mindestens drei Gruppensitzungen nahmen 114 Mütter $(88,4 \%)$ teil. Die Väter beteiligten sich nur in geringem Umfang (6,3 \%).

\section{Ergebnisse}

\subsection{Anwendung der Erziehungsstrategien unmittelbar nach dem Training}

Die Antwortkategorie „Habe ich nie ausprobiert“ wurde über alle Strategien hinweg am seltensten angekreuzt. Etwa 3/4 aller Strategien wurden von weniger als $10 \%$ der Eltern „nie“ ausprobiert (alle positiven Strategien außer Punktekarte und aus Sitzung 3 Direktes Ansprechen, Absichtliches Ignorieren, Klare, ruhige Anweisungen, Logische Konsequenzen). Etwa jedes fünfte bis sechste Elternteil benutzte niemals die Strategien Auszeit, Kooperationsroutine, Routine zur Verhaltensänderung und Aktivitätenpläne. $23 \%$ der Eltern setzte nie die Strategie Punktekarte ein. Am seltensten kam der Stille Stuhl zum Einsatz. 33 \% der Eltern wendeten diese Strategie nicht an.

Die meisten Strategien wurden bereits vor dem Programm von einem Großteil der Eltern praktiziert; z. B. benutzten über $3 / 4$ der Eltern die Strategien Mit Kind sprechen $(76,7 \%)$ und Zuneigung zeigen $(78,1 \%)$ schon vor dem Training. Die Strategien Wertvolle Zeit, Beschreibendes Lob, Aufmerksamkeit schenken, Spannende Aktivitäten und Beiläufiges Lernen wurden immerhin von mehr als der Hälfte schon vor dem Training verwendet. Nur wenige Eltern setzten bereits vor dem Programm die 
Punktekarte (10,0 \%) und den Stillen Stuhl (8,2 \%) ein. Es fällt auf, dass die Strategien zur Förderung der kindlichen Entwicklung (Sitzung 2) von den Eltern insgesamt am häufigsten schon vor der Teilnahme am Elterntraining praktiziert worden waren.

Die Antwortkategorie „Habe ich während des Programms ausprobiert“ kreuzten zwischen 3,9\% und 43,4 \% der Eltern an. Am häufigsten wurden die Routine zur Verhaltensänderung (43,4 \%), Kooperationsroutine (41,1\%) und Punktekarte (40,0\%) während des Programms ausprobiert. Hingegen wurden Mit Kind sprechen (3,9\%), Zuneigung zeigen (6,9 \%), Beschreibendes Lob (7,8 \%) und Aufmerksamkeit schenken $(9,4 \%)$ von Eltern nur selten während des Trainings zum ersten Mal ausprobiert: wie oben beschrieben hatten allerdings viele Eltern diese Strategien bereits praktiziert.

Es gaben zwischen $16,7 \%$ und 54,5\% der Eltern an, eine Strategie „als festen Bestandteil in die Erziehung aufgenommen“ zu haben. Die Strategien Klare, ruhige Anweisungen (54,5 \%), Logische Konsequenzen (48,4 \%) und Direktes Ansprechen $(42,6 \%)$ wurden dabei am häufigsten genannt. Die Strategien Lernen am Modell (16,7 \%) und Aktivitätenpläne (18,4\%) wurden am seltensten beibehalten.

\subsection{Anwendung der Erziehungsstrategien bei der 1-Jahres-Nacherhebung}

Für die nachfolgende Beschreibung wurden die Kategorien „selten“ und „manchmal“ zu „unregelmäßig“ zusammengefasst, ebenso die Kategorien „oft“ und „sehr oft“ zu „regelmäßig“. Einige Strategien wurden von bis zu 99 \% der Eltern regelmäßig angewandt, während andere von bis zu $63 \%$ nie ausprobiert wurden.

Fast die Hälfte aller Strategien wurden von (nahezu) allen Eltern benutzt (Wertvolle Zeit, Mit Kind sprechen, Zuneigung zeigen, Beschreibendes Lob, Aufmerksamkeit schenken, Spannende Aktivitäten, Beiläufiges Lernen, Direktes Ansprechen und Logische Konsequenzen). Dies betraf - mit Ausnahme der Punktekarte - hauptsächlich Strategien zur Förderung der kindlichen Entwicklung. Die Strategien Lernen am Modell, Familienregeln, Kooperationsroutine und Routine zur Verhaltensänderung wurden von mehr als $90 \%$ der Eltern mindestens „selten“ eingesetzt. Während etwa jeder vierte bis zehnte die Strategien Fragen-Sagen-Tun, Absichtliches Ignorieren, Auszeit und Aktivitätenpläne nicht einsetzte, traf dies bezüglich der Strategie Punktekarte (55,9\%) und Stiller Stuhl (63,1 \%) für über $50 \%$ der Eltern zu.

Viele der Strategien - hauptsächlich jene zum Umgang mit Problemverhalten wurden von über der Hälfte der Eltern „unregelmäßig“ (zwischen 1x pro Monat und 1x pro Woche) angewandt: Absichtliches Ignorieren (51,4 \%), Auszeit (59,1\%), Kooperationsroutine (61,9\%) und Routine zur Verhaltensänderung $(63,9 \%)$. Bei Sitzung 2 traf dies für die Strategie Lernen am Modell (55,5\%) zu und bei Sitzung 4 für Aktivitätenpläne (56,9 \%). Die Strategien Mit Kind sprechen (1,8 \%), Zuneigung zeigen (0,9 \%), Beschreibendes Lob (2,7 \%) und Aufmerksamkeit schenken (3,6 \%) verwendeten weniger als $5 \%$ der Eltern unregelmäßig.

Zum Zeitpunkt FU1 praktizierten zwischen 6,3\% und 99,1 \% der Eltern eine Strategie „regelmäßig“. Die favorisierten Strategien waren mit über 90 \% Mit Kind 
sprechen (98,2 \%), Zuneigung zeigen (99,1 \%), Beschreibendes Lob (97,3\%), Aufmerksamkeit schenken (96,4 \%) und Direktes Ansprechen (90,9 \%). Die folgenden Erziehungsstrategien benutzen weniger als $15 \%$ der Eltern regelmäßig: Punktekarte (6,3\%), Stiller Stuhl (11,7 \%) und Auszeit (12,7 \%).

\subsection{Vergleich der Anwendungshäufigkeit der Erziehungsstrategien}

Der Vergleich der Strategien erfolgte über die Bildung von Rangreihen zu den beiden Messzeitpunkten nach dem Kriterium der Anwendungshäufigkeit. Da die Anwendung der Strategien mit unterschiedlichen Skalen erhoben wurden, mussten vergleichbare Kategorien erstellt werden. Zu diesem Zweck wurden die Antwortkategorien „Schon vorher praktiziert" und „Fester Bestandteil“ (Post) und die Kategorien „Manchmal“, „Oft“ und „Sehr oft" für den Zeitpunkt FU1 zusammengefasst.

Die Eltern wandten direkt nach dem Training am häufigsten Strategien aus Sitzung 2 an. Die Strategien Stiller Stuhl, Punktekarte, Kooperationsroutine und Routine zur Verhaltensänderung wurden dagegen nur relativ selten benutzt. Die Rangreihe der Strategien zum Zeitpunkt FU1 ergab ein sehr ähnliches Bild. Bis auf die Strategien Direktes Ansprechen (Post: 7 - FU1: 1), Absichtliches Ignorieren (Post: 11 - FU1: 15) und Auszeit (Post: 13 - FU1: 18) ergaben sich keine Rangplatzwechsel von mehr als drei Positionen. Dieser Eindruck wird durch die statistische Analyse bestätigt. Die Korrelation der beiden Rangreihen betrug $r=.93(p<.01)$.

\subsection{Anwendung der Erziehungsstrategie „Auszeit"}

Im Kursbeurteilungsbogen wurde erfragt, in welchen Situationen die Eltern die „Auszeit" anwenden. Zeigte das Kind trotz wiederholter Aufforderungen keine Reaktion, so verhängte fast jede zweite Familie eine „Auszeit“ (48,9\%). Bei Wutanfällen und Schreien setzte knapp ein Drittel der Eltern (28,9\%) die "Auszeit“ ein. Jeder Vierte (25,6 \%) benutzte diese Strategie, wenn es zu Streit zwischen Geschwistern kam. Verhielt sich ein Kind aggressiv, musste es bei 17,8 \% der Eltern in die „Auszeit“. 14,4 \% der Eltern setzten sie dann ein, wenn der "Stille Stuhl“ keine Wirkung zeigte. Fast eben so viele (13,3\%) praktizierten die „Auszeit", wenn es keine andere Möglichkeit bzw. logische Konsequenz gab. Um sich selbst zu beruhigen, schickten 8,9\% der Eltern die Kinder in die „Auszeit“. Etwa $4 \%$ sehen die Strategie als Vermeidung einer Eskalation an.

Um die Anwendungshäufigkeit der Strategie „Auszeit“ bezüglich der drei Messzeitpunkte miteinander vergleichen zu können, wurde auf Grundlage des Kursbeurteilungsfragebogens und des Strategiebogens (FU1) eine Schätzung der absoluten Anwendungshäufigkeit pro Monat vorgenommen: einmal pro Monat („selten“), viermal pro Monat („manchmal“), zwölfmal pro Monat („oft“) und 30mal pro Monat („sehr oft"). Es ergibt sich beim Prä-Zeitpunkt pro Monat ein durchschnittlicher Wert von 5,3 pro Familie. Unmittelbar nach dem Training wendeten Eltern durchschnittlich 5,1-mal die „Auszeit“ an. Eine starke Abnahme der Verwendung ist bei 
der 1-Jahres-Nacherhebung zu verzeichnen. Die „Auszeit" wurde durchschnittlich nur noch 2,9-mal im Monat praktiziert. Die einfaktorielle Varianzanalyse bestätigt, dass es zu einer statistisch relevanten Abnahme der Auszeitanwendung über die drei Messzeitpunkte hinweg $\operatorname{kam}(F(1.98)=14.52 ; p<.01)$.

\section{Diskussion}

Die Ergebnisse zeigen, dass ein Großteil der Eltern vor allem die positiven Strategien - mit Ausnahme der komplexeren Strategien „Fragen-Sagen-Tun“ und „Punktekarte“ - bereits vor dem Training verwendet hatten. Die Erziehungsstrategien zum Umgang mit Problemverhalten waren im Vergleich dazu vor dem Training relativ unbekannt. Keine dieser Strategien wurde vorher von mehr als der Hälfte der Eltern ausprobiert. Die Vorliebe der Eltern für die positiven Strategien blieb auch bestehen, denn sowohl zu Post als auch zum Zeitpunkt FU1 stammen die fünf häufigsten Strategien aus der Sitzung 2.

Diese Tatsachen sprechen gegen die These, dass es durch die Triple P-Teilnahme $\mathrm{zu}$ einer Verschiebung von positiven zu negativen Strategien kommt (Deegener $\mathrm{u}$. Hurrelmann, 2002). Generell scheint das Erziehungsverhalten relativ stabil zu sein, da sich die Beliebtheitsreihenfolge der Strategien nicht änderte. Die Vorgehensweise von Triple P betont den Einsatz unterstützender und fördernder Strategien. Zum Zeitpunkt FU1 werden sieben positive Strategien von über $90 \%$ der Eltern verwendet. Deshalb beschreibt der Begriff „Positives Erziehungsprogramm“ dieses Präventionsprogramm auch in diesem Sinne sehr zutreffend. Der Rückschluss, dass der Schwerpunkt von Triple P auf Disziplinierungsmaßnahmen liegt (Tschöpe-Scheffler, 2003) oder auf Strafe und Gehorsam basiert (Deegener u. Hurrelmann, 2002), ist auf der Grundlage der vorliegenden Ergebnisse nicht gerechtfertigt.

Generell waren die Eltern sehr offen und brachten eine hohe Bereitschaft mit, die Erziehungsstrategien auszuprobieren. Es kommen unterschiedliche Ursachen in Betracht, warum die Strategien Spannende Beschäftigungen, Lernen am Modell, Fragen-Sagen-Tun, Punktekarte, Familienregeln, Absichtliches Ignorieren, Stiller Stuhl, Erziehungsroutinen und Aktivitätenpläne von vielen Eltern nicht weiter eingesetzt wurden. Mögliche Gründe sind, dass diese als zu aufwändig empfunden wurden, der Sinn nicht klar verständlich war oder die gewünschten Erfolge schlichtweg ausblieben. Da für den deutschen Sprachraum hiermit erstmals Daten zum Einsatz der Erziehungsstrategien vorgelegt werden, ist ein Vergleich mit der Literatur nicht möglich.

Die Strategien Punktekarte, Familienregeln, Absichtliches Ignorieren und Stiller Stuhl wurden im Vergleich zu anderen Strategien seltener angewandt. Diese offenkundig geringere Akzeptanz könnte zum einen darauf zurückzuführen sein, dass hauptsächlich Eltern von verhaltensauffälligen Kindern den Einsatz als sinnvoll erachteten. Da die Daten an einer universellen Stichprobe erhoben wurden, war die Varianz an Ver- 
haltensauffälligkeiten eingeschränkt. Eine weitere Möglichkeit wäre, dass bereits der Einsatz der positiven Strategien die o.g. Strategien „überflüssig“ machte.

Auszeitverfahren wurden vergleichsweise selten angewendet, da sie hauptsächlich bei schwerwiegendem kindlichem Problemverhalten eingesetzt werden sollten. Dieses trat jedoch in der universellen Stichprobe relativ selten auf. Durch den gezielten Einsatz anderer Triple P-Strategien sollte außerdem die Verwendung der Auszeitverfahren möglichst überflüssig werden (Fröhlich, 2004). Die vorliegenden Ergebnisse scheinen genau dies zu bestätigen. Es wurde über die einzelnen Messzeitpunkte hinweg eine signifikante Reduktion der Anwendungshäufigkeit festgestellt. Das lässt die Annahme zu, dass Eltern lernen, die „Auszeit“ gezielt in schwerwiegenden Situationen anzuwenden. Deswegen ist die Befürchtung, dass die Teilnahme an Triple $\mathrm{P}$ zu einer ,inflationären Anwendung“ der Auszeitverfahren und zu einem Einsatz bei „alltäglichen Problemen“ führen könnte (Deegener u. Hurrelmann, 2002), unbegründet. Das Gegenteil scheint der Fall zu sein.

Es muss bei der Interpretation der Ergebnisse berücksichtigt werden, dass sie sich aufgrund der geringeren Teilnahme sozial benachteiligter Familien nicht auf die Allgemeinbevölkerung übertragen lassen. Wenn jedoch sozial benachteiligte Familien für Präventionsangebote gewonnen werden können, äußern sie sich genauso zufrieden mit dem Training und nehmen gleichermaßen teil wie sozial besser gestellte Familien (Heinrichs et al., 2006b). Ob auch die eingesetzten Erziehungsstrategien identisch sind, muss in zukünftigen Analysen überprüft werden.

Inzwischen hat sich Triple P in mehreren Ländern zu einem umfassenden mehrstufigen Unterstützungssystem in Erziehungs- und Familienfragen entwickelt und es ist zu hoffen, dass auch im deutschsprachigen Raum Triple P allen Eltern zur Verfügung gestellt werden kann, die von diesem Angebot profitieren möchten (Cina et al., 2006).

\section{Literatur}

Bandura, A. (1977). Self-efficacy: Toward a unifying theory of behavioral change. Psychological Review, 84, 191-215.

Bertram, H., Heinrichs, N., Kuschel, A., Kessemeier, Y., Saßmann, H., Hahlweg, K. (2003). Projekt „Zukunft Familie“. Erste Ergebnisse der Rekrutierung. Verhaltenstherapie und Verhaltensmedizin, 24, 187-204.

Bundesministerium für Familie, Senioren, Frauen und Jugend (BMFSFJ) (2003). Gewaltfreie Erziehung. Eine Bilanz nach Einführung des Rechts auf gewaltfreie Erziehung 2003 (Broschüre). Online im Internet (eingestellt: 2004-03-22): URL: http://www.bmfsfj.de/Kategorien/Publikationen/Publikationen,did=17396.html (2004-09-14).

Cina, A., Bodenmann, G., Hahlweg, K., Dirscherl, T., Sanders, M. R. (2006). Triple P (Positive Parenting Program): Theoretischer und empirischer Hintergrund und erste Erfahrungen im deutschsprachigen Raum. Zeitschrift für Familienforschung, 18, 66-88.

Deegener, G., mit Unterstützung von Hurrelmann, K. (2002). Kritische Stellungnahme zu Triple P. Unveröffentlichtes Manuskript, Universität Homburg, Universitäts-Nervenklinik, Abteilung für Kinder und Jugendliche, Homburg/Saar.

(c) Vandenhoeck \& Ruprecht GmbH \& Co. KG, Göttingen 2007 
Fröhlich, U. (2004). Fachliche Gegendarstellung zur „Kritischen Stellungnahme“ gegenüber Triple P. Unveröffentlichtes Manuskript, Halle.

Greenberg, M. T., Domitrovich, C., Bumberger, B. (2001). The prevention of mental disorders in school-aged children: Current state of the field. Prevention \& Treatment 4: np.

Hahlweg, K. (2001). Bevor das Kind in den Brunnen fällt: Prävention von kindlichen Verhaltensstörungen. In W. Deutsch, M. Wenglorz (Hrsg.), Zentrale Entwicklungsstörungen bei Kindern und Jugendlichen (S. 189-241). Stuttgart: Klett-Cotta.

Hahlweg, K., Heinrichs, N., Bertram, H., Kuschel, A., Widdecke, N. (in Druck). Körperliche Bestrafung: Prävalenz und Einfluss auf die psychische Entwicklung bei Vorschulkindern. Kindheit und Entwicklung.

Hart, B., Risley, T. R. (1995). Meaningful differences in the everyday experience of young american children. Sydney: Paul H. Brooks.

Heinrichs, N. (2006). The effects of two different incentives on recruitment rates of families into a selective prevention program. Journal of Primary Prevention, 27, 345-365.

Heinrichs, N., Bertram, H., Kuschel, A., Hahlweg, K. (2005). Parent recruitment and retention in a universal prevention program for child behavior and emotional problems. Prevention Science, 6, 275-286.

Heinrichs, N., Hahlweg, K., Döpfner, M. (2006a). Familien stärken: Evidenz-basierte Ansätze zur Unterstützung der psychischen Gesundheit von Kindern. Münster: Verlag für Psychotherapie.

Heinrichs, N., Krüger, S., Guse, U. (2006). Der Einfluss von Anreizen auf die Rekrutierung von Eltern und auf die Effektivität eines präventiven Elterntrainings. Zeitschrift für Klinische Psychologie und Psychotherapie, 35, 97-108.

Heinrichs, N., Hahlweg, K., Bertram, H., Kuschel, A., Naumann, S., Harstick, S. (2006b). Die langfristige Wirksamkeit eines Elterntrainings zur universellen Prävention kindlicher Verhaltensstörungen: Ergebnisse aus der Sicht der Mütter und Väter. Zeitschrift für Klinische Psychologie und Psychotherapie, 35, 82-96.

Heinrichs, N., Hahlweg, K., Kuschel, A., Krüger, S., Bertram, H., Harstick, S., Naumann, S. (2006c). Triple P aus der Sicht der Eltern. Kindheit und Entwicklung, 15, 19-26.

Kuschel, A., Lübke, A., Köppe, E., Miller, Y., Hahlweg, K., Sanders, M. R. (2004). Häufigkeit psychischer Auffälligkeiten und Begleitsymptome bei drei- bis sechsjährigen Kindern: Ergebnisse der Braunschweiger Kindergartenstudie. Zeitschrift für Kinder- und Jugendpsychiatrie und Psychotherapie, 32, 97-106.

Lösel, F., Beelmann, A., Jaursch, S., Koglin, U., Stemmler, M. (2005). Entwicklung und Prävention früher Probleme des Sozialverhaltens: Die Erlangen-Nürnberger Studie. In M. Cierpka (Hrsg.), Möglichkeiten der Gewaltprävention (S. 201-229). Göttingen: Vandenhoeck \& Ruprecht.

Lübke, A., Miller, Y., Köppe, E., Kuschel, A., Hahlweg, K. (2000). Abschlussbericht über die Braunschweiger Kindergartenstudie. Braunschweig: Christoph-Dornier-Stiftung für Klinische Psychologie.

Offord, D. R., Boyle, M. H., Szatmari, P., Rae-Grant, N. I., Links, P. S., Cadman, D. T., Byles, J. A., Crawford, J. W., Blum, H. M., Byrne, C., Thomas, H., Woodward, C. A. (1987). Ontario Child Health Study II. Six-month prevalence of disorder and rates of service utilization. Archives of General Psychiatry, 44, 832-836.

Patterson, G. R. (1982). Coercive family process. Eugene, OR: Castalia.

Perrez, M., Moggi, F. (1990). Materialien zur Epidemiologie der Gewalt in der Familie und den Kurz- und Langzeitwirkungen. In Pro Familia (Hrsg.), Familie und Gewalt (S. 16-31). Bern. 
Petermann, U., Petermann, F. (2006). Themenschwerpunkt Erziehungskompetenz. Kindheit und Entwicklung, 15, 1-8.

Prinz, R. J., Sanders, M. R. (2006).Testing effects on parenting at a broad scale: The U.S. Triple P System Population Trail. In N. Heinrichs, K. Hahlweg, M. Döpfner (Hrsg.), Strengthening families: Evidence-based approaches to support child mental health (S. 485-511). Münster: Verlag für Psychotherapie.

Rutter, M. (1989). Pathways from childhood to adult life. Journal of Child Psychology and Psychiatry, 30, 23-51.

Sanders, M. R. (1996). New directions in behavioral family intervention with children. In T. H. Ollendick, R. J. Prinz (Hrsg.), Advances in clinical child psychology (S. 283-330). New York: Plenum Press.

Sanders, M. R. (1999). The Triple P-Positive Parenting Program: Towards an empirically validated multi-level parenting and family support strategy for the prevention and treatment of child behavior and emotional problems. Child and Family Psychology Review, 2, 71-90.

Sanders, M. R. (2006). Making a population approach to positive parenting really work. In N. Heinrichs, K. Hahlweg, M. Döpfner (Hrsg.), Strengthening families: Evidence-based approaches to support child mental health (S. 45-76). Münster: Verlag für Psychotherapie.

Sanders, M. R., Markie-Dadds, C., Turner, K. (2003). Theoretical, scientific and clinical foundations of the Triple P -Positive Parenting Program: A population approach to the promotion of parenting competence. Parenting Research and Practice Monograph, 1, 1-21.

Schneewind, K. (1999). Familienpsychologie. 2. Auflage. Göttingen: Hogrefe.

Tschöpe-Scheffler, S. (2003). Elternkurse auf dem Prüfstand. Opladen: Leske \& Budrich.

Korrespondenzadressen: Dr. Annett Kuschel, Institut für Rehabilitationswissenschaften, Humboldt-Universität zu Berlin, Georgenstraße 36, 10117 Berlin.

E-Mail: kuschela@staff.hu-berlin.de

Prof. Dr. Kurt Hahlweg, Institut für Psychologie, Technische Universität Braunschweig, Spielmannstraße 12a, 38106 Braunschweig. E-Mail: k.hahlweg@tu-bs.de

Dipl.-Psych. Sandra Beslmeisl und Prof. Dr. Martin Hautzinger, Eberhard Karls Universität Tübingen, Christophstr. 2, 72072 Tübingen, E-Mail: sandra.beslmeisl@web.de, hautzinger@uni-tuebingen.de 